\title{
PRODUTIVIDADE E LONGEVIDADE DE CRISÂNTEMOS, CULTIVADOS EM HIDROPONIA, EM RESPOSTA A DOSES DE CÁLCIO
}

\author{
Chrysanthemum productivity and longevity in a hydroponic \\ system in response to calcium doses \\ Maurício Soares Barbosa ${ }^{1}$, José Geraldo Barbosa ${ }^{2}$, Fernando Luiz Finger ${ }^{1}$, \\ Telma pontes ${ }^{3}$, Mariana Rubim Raimundo ${ }^{1}$, Thiago Costa Ferreira ${ }^{3}$
}

\begin{abstract}
RESUMO
Para verificar a resposta de diferentes variedades de crisântemos a doses de cálcio um experimento foi conduzido em casa de vegetação sob sistema hidropônico de três fases e cascalho como substrato. Utilizou-se o delineamento em blocos casualizados com parcelas subdivididas, sendo as doses de cálcio $\left(1,0 ; 2,5 ; 4,0 ; 5,5 \mathrm{mmol} \mathrm{L}^{-1}\right)$ dispostas nas parcelas e três variedades (Blush Hawai, Calabria e Dark Flamengo) nas subparcelas, com 3 repetições. Para as diferentes doses de cálcio, utilizou-se solução nutritiva completa. Foram avaliados o peso da matéria fresca da folha, caule, inflorescência e parte aérea, ciclo, comprimento de haste e número e longevidade das inflorecencias, expressa pelo número de dias da colheita ao descarte das mesmas (murcha e perda de coloração das flores). As variedades Calabria e Blush Hawai apresentaram-se mais precoces e mais produtivas. Houve aumento linear na longevidade das três variedades com o aumento das concentrações de cálcio na solução nutritiva. O melhor desenvolvimento e crescimento das variedades de crisântemos foi obtido quando utilizada a solução nutritiva com $4,0 \mathrm{mmol} \mathrm{L}^{-1}$ de cálcio.
\end{abstract}

Termos para indexação: Dendranthema grandiflora (Ramat.) Kitam., produção, sistema hidropônico, vida pós-colheita.

\begin{abstract}
To verify the response of different varieties of chrysanthemum to doses of calcium an experiment was conducted in a green house using a three-phase hydroponic system on a gravel substrate. It was set up in a completely random blocks with split-plot design, using four calcium doses $\left(1.0 ; 2.5 ; 4.0 ; 5.5 \mathrm{mmol} \mathrm{L}^{-1}\right)$ in the plots disposed and three chrysanthemum varieties (Blush Hawai, Calabria and Dark Flamengo) in the subplots. Three repetitions were used with 96 plants by repetition. Complete nutrient solutions were used with the different calcium doses. The following factors were evaluated: fresh matter weight of leaf, stem, inflorescence and shoot; cycle; number of inflorescences, stem length and the longevity, expressed in the number of days from harvest to disposal of the inflorescences (withered and losing flower coloring). The earliest and most productive varieties were Calabria and Blush Hawai. There was linear increase in the longevity of the three varieties with the increase of the concentrations of calcium in the nutrient solution. The best development and growth of the varieties of chrysanthemum was obtained when used the nutrient solution with $4.0 \mathrm{mmol} \mathrm{L}^{-1} \mathrm{calcium}$.
\end{abstract}

Index terms: Dendranthema grandiflora (Ramat.) Kitam., production, hydroponics system, postharvest.

(Recebido em 28 de dezembro de 2007 e aprovado em 1 de julho de 2008)

\section{INTRODUÇÃO}

O mercado de floricultura atual está numa situação privilegiada, por causa dos consumidores mais exigentes, os produtores estão buscando novidades e tecnologia e, dessa maneira, sendo mais competitivos entre si. Outro ponto favorável ao crescimento do setor é a aproximação do produtor com o pesquisador em busca de tecnologias adaptadas às condições climáticas brasileiras e regionais, visando a maior eficiência e qualidade na produção de flores, tais como, produtos mais resistentes, melhoria nos sistemas de adubação, irrigação e fertilização. Em 2007, o setor de floricultura no Brasil, movimentou R \$ 2,4 bilhões de reais. $\mathrm{O}$ mercado é composto por 7 mil produtores, que atuam em 25 mil pontos de venda (Brasil, 2008). No estado de Minas Gerais a produção de flores de corte é uma atividade realizada por 188 produtores, numa área plantada de aproximadamente 290,6836 ha. A produção é vendida para todo o Brasil e também exportada para países da Europa, Ásia e América do Norte (Landgraf e Paiva, 2009).

O Crisântemo para flor de corte é a segunda espécie mais comercializada no Brasil, ficando apenas atrás da rosa. Seu cultivo é feito em canteiros, empregando-se misturas de solo e matéria orgânica, onde se realizam adubações concentradas, buscando-se o máximo de qualidade e rendimento. Nos leitos de cultivo é comum ocorrerem problemas como perdas de adubo,

${ }^{1}$ Universidade Federal de Viçosa/UFV - Departamento de Fitotecnia/DFT - Viçosa, MG

${ }^{2}$ Universidade Federal de Viçosa/UFV - Departamento de Fitotecnia/DFT - Centro de Ciências Agrárias - Avenida P. H. Rolfs - s/n - Centro - Campus

Universitário - 36570-000 - Viçosa, MG - jgeraldo@ufv.br

${ }^{3}$ Universidade Federal de Viçosa/UFV - Viçosa, MG 
salinização e infestação progressiva de microorganismos do solo. (Barbosa et al., 2000). Além desses problemas, o ciclo curto, alto retorno financeiro e o aumento na qualidade e vida pós-colheita sugerem o uso da técnica do cultivo hidropônico em floricultura, particularmente para o crisântemo (Hansen, 1999).

Os fatores nutricionais pré-colheita, bem como ambientais, estão diretamente relacionados com maior produtividade e vida pós-colheita das plantas (Roude et al., 1991). Poucos estudos relatam a relação entre nutrição e pós-colheita para plantas de crisântemos, principalmente, em relação ao cálcio. $\mathrm{O}$ cálcio está envolvido em vários processos durante o crescimento e senescência das plantas, pois influencia a estrutura e função da membrana e parede celular (Halevy et al., 2001), estabilizando a membrana celular pela ligação entre fosfatos e grupos carboxílicos de fosfolipídios e proteínas. Em tecidos deficientes em cálcio o prejuízo da integridade da membrana conduz ao aumento na velocidade da respiração e da degradação líquida de proteínas e clorofila, levando à senescência (Taiz \& Zeiger, 2003).

Segundo Gislerod (1999), o cálcio tem importante papel na qualidade, no crescimento e desenvolvimento de plantas ornamentais e sua deficiência está relacionada a vários fatores, como necroses em poinsettia, suscetibilidade da rosa ao Botrytis, dureza do caule de cravos e longevidade pós-colheita de flores. Dessa forma, pode-se relacionar as funções do cálcio, particularmente a manutenção da estabilidade e funções da membrana celular e o estímulo em alguns eventos metabólicos, com a regulação do crescimento e vida pós-colheita das plantas, mostrando sua importância no crescimento e vida de vaso das flores cortadas. Assim, os objetivos do experimento foram avaliar a produção, qualidade e longevidade póscolheita de inflorescências de crisântemos, cultivados em sistema hidropônico, sob diferentes doses de cálcio.

\section{MATERIAL E MÉTODOS}

O experimento foi conduzido no Setor de Floricultura, do Departamento de Fitotecnia da Universidade Federal de Viçosa, sob condições de casa de vegetação. Utilizou-se o modelo em blocos casualizados com parcelas subdivididas, sendo as 4 doses de cálcio $\left(1,0 ; 2,5 ; 4,0 ; 5,5 \mathrm{mmol} \mathrm{L}^{-1}\right)$ dispostas nas parcelas e 3 variedades de crisântemos nas subparcelas com 3 repetições. Para as diferentes doses de cálcio utilizou-se solução nutritiva recomendada por Barbosa (2003) modificada com 11,51;2,88; 1,95; 12,92; 1,0; $2,44 \mathrm{mmol} \mathrm{L}^{-1}$ de N-NO ${ }_{3}^{-}, \mathrm{N}_{-} \mathrm{NH}_{4}^{+}, \mathrm{P}_{-} \mathrm{H}_{2} \mathrm{PO}_{4}^{-}, \mathrm{K}^{+}, \mathrm{Mg}^{2+}, \mathrm{S}-$ $\mathrm{SO}_{4}^{-2}$ e $30 ; 5 ; 50 ; 40 ; 0,1 ; 2 \mu \mathrm{mol} \mathrm{L}^{-1}$ de B, Cu, Fe, Mn, Mo, $\mathrm{Zn}$, respectivamente. Foram utilizadas as variedades do grupo decorativo Blush Hawai (rosa), Calabria (branca) e Dark Flamengo (roxa).

O cultivo foi realizado em bancadas de amianto de 6,60 m de comprimento x $0,84 \mathrm{~m}$ de largura $\mathrm{x} 0,18 \mathrm{~m}$ de profundidade. No leito de cultivo, utilizou-se o espaçamento de $12 \times 10 \mathrm{~cm}$, sendo que cada unidade experimental continha 6 fileiras de plantas (duas por variedade) com 16 plantas por fileira. Além das 6 fileiras procedeu-se o plantio nas laterais das bancadas, cujas plantas funcionaram como bordaduras.

Para o crescimento vegetativo, as plantas foram mantidas sob 40 dias longos (DL) com 16 horas de luz, sendo 4 horas por meio de luz artificial. Após esse período, as plantas foram mantidas sob 35 dias curtos (DC), para indução ao florescimento, cobrindo-se as bancadas de plantio com uma lona preta das 17 às 7 horas. Após a indução, eliminou-se o botão principal e as plantas foram mantidas sob dias normais até a data da colheita (2/3 das inflorescências abertas). Durante o experimento, foi realizado tutoramento, para evitar tombamento das plantas.

A circulação das soluções nutritivas foi controlada por temporizador, que acionava o sistema por 15 minutos, a cada intervalo de 1 hora, das 7 às 19 horas. No período noturno, o sistema foi acionado por 15 minutos, a cada 3 horas. A reposição do volume de água foi efetuada quando o volume das soluções atingiu $90 \%$ do total do reservatório de fibra de vidro $(900 \mathrm{~L})$. O pH e a condutividade elétrica (CE) foram monitorados diariamente. A correção do $\mathrm{pH}$ foi feita para valores entre 5,5 a 6,5, utilizando-se soluções de $\mathrm{NaOH} 1 \mathrm{~mol} \mathrm{~L}^{-1}$ ou de $\mathrm{HCl} 1 \mathrm{~mol} \mathrm{~L}^{-1}$, conforme a necessidade. A reposição dos nutrientes foi realizada quando a $\mathrm{CE}$ atingiu $70 \%$ do valor inicial.

Plantas da primeira fileira para cada variedade foram coletadas quando $2 / 3$ das inflorescências estavam abertas e foram avaliados: peso da matéria fresca da folha, do caule, da inflorescência e da parte aérea; ciclo (plantio até 2/3 das inflorescências abertas); número de inflorescências entre 1 e $3 \mathrm{~cm}$; maiores que $3 \mathrm{~cm}$, número total de inflorescências e comprimento de haste. Para avaliação da longevidade, expressa pelo número de dias da colheita ao descarte das inflorescências, plantas da segunda fileira foram coletadas e as hastes colocadas em vaso com água em sala à temperatura ambiente, a qual variou de 18 a $25^{\circ} \mathrm{C}$. O descarte foi realizado quando $50 \%$ ou mais das inflorescências estavam senescentes, ou seja, sem aspecto decorativo,em virtude da murcha e necrose das peças florais.

Os dados obtidos foram submetidos à análise de variância e de regressão, sendo as médias testadas pelo teste de Newman keuls a 5\% de probabilidade, para os tratamentos qualitativos. Para os tratamentos quantitativos (doses de 
cálcio) foram ajustados modelos de regressão, sendo selecionadas dentro das equações de regressão, aquelas de efeito significativo, pelo teste $\mathrm{F}$, a 1 e $5 \%$ de probabilidade e de maior soma de quadrado, ou seja, maior $\mathrm{R}^{2}$.

\section{RESULTADOS E DISCUSSÃO}

Para produção de matéria fresca das folhas, as variedades Calabria e Blush Hawai não diferiram entre si e foram superiores à Dark Flamengo. Observou-se o mesmo comportamento para produção de matéria fresca do caule, da inflorescência e da parte aérea (Tabela 1). Os resultados mostram a superioridade das variedades Calabria e Blush Hawai em relação à Dark Flamengo, característica importante na padronização e comercialização do crisântemo. Barbosa et al. (2000), estudando crisântemo de corte 'Yellow Polaris' cultivado em sistema hidropônico, encontrou valores médios para produção de matéria fresca da parte aérea entre 45,85 e 60,45 g por planta, valores estes inferiores aos encontrados neste trabalho. Segundo Muniz et al. (2009), é desejável que os valores de matéria fresca sejam elevados, o que pode representar plantas fortes e consistentes, e, no caso de flores cortadas, maior vida pós-colheita. As diferentes doses de cálcio não afetaram significativamente a produção de matéria fresca da parte aérea.

As variedades Blush Hawai e Calabria com ciclo estimado de 92,5 e 93,2 dias, respectivamente, foram mais precoces que a Dark Flamengo, cujo ciclo estimado foi de 98,1 dias (Tabela 2). Segundo Barbosa (2003), o ciclo para crisântemos de corte cultivados em sistema convencional varia de 95 a 115 dias. O ciclo constitui um ponto crucial na produção, particularmente para datas específicas como dia das mães, namorados, finados, Natal, entre outros, sendo a precocidade importante fator de redução de custos. Todas as variedades mostraram-se mais precoces em relação à variedade para corte de flor 'Yellow Polaris', cujo ciclo foi de 110 dias cultivada em sistema hidropônico, como observado por Barbosa et al. (2000).

O comportamento para o ciclo em função das doses de cálcio pode ser visualizado na Figura 1, sendo o modelo de regressão linear o que melhor se ajustou aos dados para as variedades Dark Flamengo e Calabria, sendo que, para a variedade Blush Hawai, não houve efeito significativo. Houve decréscimo no ciclo à medida que se aumentaram as doses de cálcio para as variedades Dark Flamengo e Calabria.

Quanto ao número de inflorescências entre 1 e $3 \mathrm{~cm}$, maiores que $3 \mathrm{~cm}$ e totais por planta, as variedades Calabria e Blush Hawai foram estatisticamente superiores à Dark Flamengo (Tabela 2). Maior número de inflorescências proporciona um maior volume e efeito visual da floração, possibilitando economia para o consumidor pelo uso reduzido de hastes em arranjos florais.

Tabela 1 - Produção de matéria fresca da folha (MFF), do caule (MFC), da inflorescência (MFI) e da parte aérea (MFPA) de variedades de crisântemos cultivados em sistema hidropônico.

\begin{tabular}{lllrr}
\hline \multirow{2}{*}{ Variedades } & \multicolumn{4}{c}{ Valores Médios $(\mathrm{g})$} \\
\cline { 2 - 5 } & MFF & MFC & MFI & \multicolumn{1}{c}{ MFPA } \\
\hline Dark Flamengo & $26,88 \mathrm{~b}$ & $35,29 \mathrm{~b}$ & $20,47 \mathrm{~b}$ & $82,65 \mathrm{~b}$ \\
Calabria & $44,38 \mathrm{a}$ & $45,30 \mathrm{a}$ & $34,42 \mathrm{a}$ & $124,10 \mathrm{a}$ \\
Blush Hawai & $50,41 \mathrm{a}$ & $43,64 \mathrm{a}$ & $36,06 \mathrm{a}$ & $130,11 \mathrm{a}$ \\
\hline
\end{tabular}

Médias seguidas da mesma letra na coluna, não diferem entre si pelo teste de Newman keuls, $5 \%$.

Tabela 2 - Número de inflorescências entre 1 e $3 \mathrm{~cm}$ (NINFE), maiores que $3 \mathrm{~cm}$ (NINFM), totais (NINFT), ciclo e comprimento de haste (COMPH) de variedades de crisântemos cultivados em sistema hidropônico.

\begin{tabular}{lccccc}
\hline \multirow{2}{*}{ Variedades } & \multicolumn{4}{c}{ Valores Médios } \\
\cline { 2 - 5 } & NINFE & NINFM & NINFT & Ciclo (dias) & COMPH (m) \\
\hline Dark Flamengo & $9,71 \mathrm{~b}$ & $3,06 \mathrm{~b}$ & $12,77 \mathrm{~b}$ & $98,1 \mathrm{~b}$ & $1,36 \mathrm{a}$ \\
Calabria & $19,24 \mathrm{a}$ & $7,47 \mathrm{a}$ & $26,71 \mathrm{a}$ & $93,2 \mathrm{a}$ & $1,28 \mathrm{~b}$ \\
Blush Hawai & $22,69 \mathrm{a}$ & $6,56 \mathrm{a}$ & $29,25 \mathrm{a}$ & $92,5 \mathrm{a}$ & $1,24 \mathrm{c}$ \\
\hline
\end{tabular}

Médias seguidas da mesma letra na coluna, não diferem entre si pelo teste de Newman keuls, $5 \%$. 


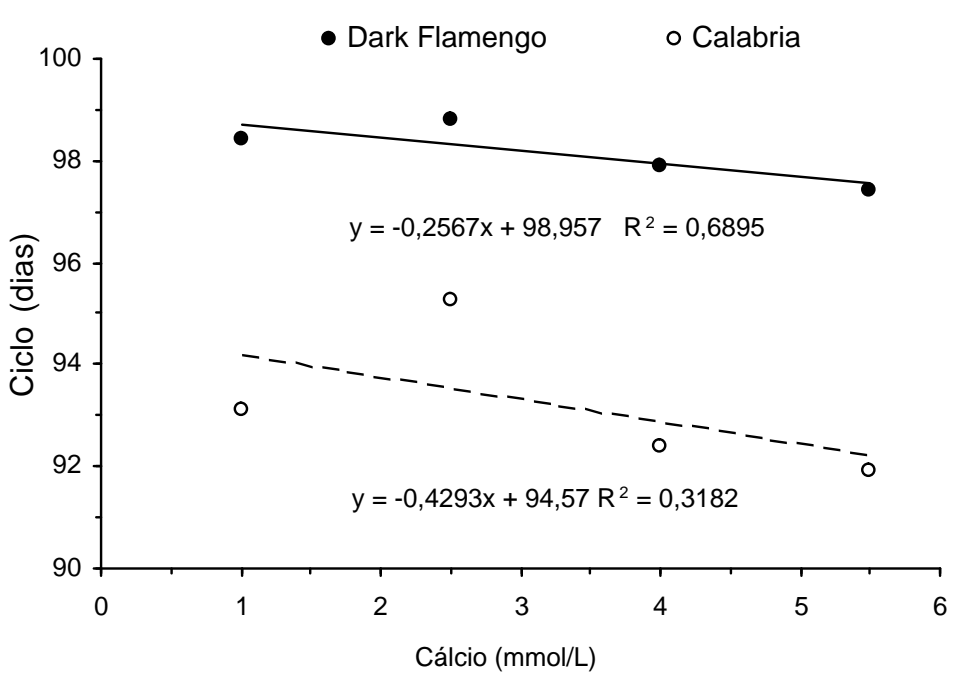

Figura 1 - Ciclo de variedades de crisântemos cultivados em sistema hidropônico em função da aplicação de doses de cálcio.

Silveira \& Minami (1999), avaliando o número de inflorescências de variedades de crisântemos para corte, do grupo decorativo, verificaram valor médio entre 5,96 e 9,79 inflorescências por planta. As diferentes doses de cálcio afetaram significativamente o número de inflorescências entre 1 e $3 \mathrm{~cm}$, como pode ser visualizado na Figura 2, sendo o modelo de regressão quadrático o que melhor se ajustou aos dados para as três variedades, observando-se um aumento no número de inflorescências com aumento na dose de cálcio, atingindo-se o ponto de máximo com as doses de 3,23, 3,96 e 4,12 $\mathrm{mmol} \mathrm{L}^{-1}$ para as variedades Calabria, Blush Hawai, e Dark Flamengo, respectivamente. Ganmore-Neumann \& Davidov (1993) estudaram o efeito de três níveis de cálcio $(1,0 ; 2,5$; 5,0 $\mathrm{mmol} \mathrm{L}^{-1}$ ) na produtividade de botões de rosa, cultivar Mercedes, em sistema de fertirrigação. Na menor dose de cálcio, o número de flores e a qualidade pós-colheita das rosas foram menores quando comparados com os demais tratamentos.

Já, para o comprimento de haste, a variedade Dark Flamengo foi superior à Calabria, e a variedade Blush Hawai foi a de menor comprimento (Tabela 2). Silveira \& Minami (1999), avaliando o comprimento de haste de cultivares de crisântemos para corte, do grupo decorativo encontraram comprimento médio de haste entre 89,9 e $95,4 \mathrm{~cm}$, sendo esses valores menores do que os encontrados para as três variedades estudadas neste trabalho. As diferentes doses de cálcio afetaram significativamente o comprimento de haste e o modelo de regressão linear foi o que melhor se ajustou aos dados para as variedades Blush Hawai e Calabria (Figura 3). Apesar da redução do comprimento da haste com o aumento das doses de cálcio, isso não afetou a qualidade comercial das hastes que é de $80-90 \mathrm{~cm}$.

De acordo com a Tabela 3, houve comportamento semelhante das variedades para longevidade nas doses de 1,0 e $2,5 \mathrm{mmol} \mathrm{L}^{-1}$ de cálcio, enquanto as variedades Calabria e Blush Hawai foram superiores à variedade Dark Flamengo quando cultivadas em solução nutritiva com as doses de 4,0 e 5,5 $\mathrm{mmol} \mathrm{L}^{-1}$ de cálcio.

O comportamento da longevidade das inflorescências em função das doses de cálcio pode ser visualizado na Figura 4, sendo o modelo linear que melhor se ajustou aos dados, onde observou-se maior longevidade com o aumento da concentração de cálcio, demonstrando o efeito do mesmo na vida pós-colheita das inflorescências de crisântemos. Segundo Taiz \& Zeiger (2003), em tecidos deficientes em cálcio o prejuízo da integridade da membrana conduz ao aumento na velocidade da respiração e na degradação líquida de proteínas e clorofila, levando à senescência. Nielsen \& Starkey (1999), cultivando a variedade de rosa híbrida Purple Parade em sistema de fertirrigação, utilizando cinco soluções nutritivas, variando doses de cálcio $(0,52 ; 1,25$; 2,50; 4,32 e 6,94 $\left.\mathrm{mmol} \mathrm{L}^{-1}\right)$, observaram que as maiores doses proporcionaram rosas de melhor qualidade, com menor porcentagem de flores murchas e menor número de abscisão de folhas. 


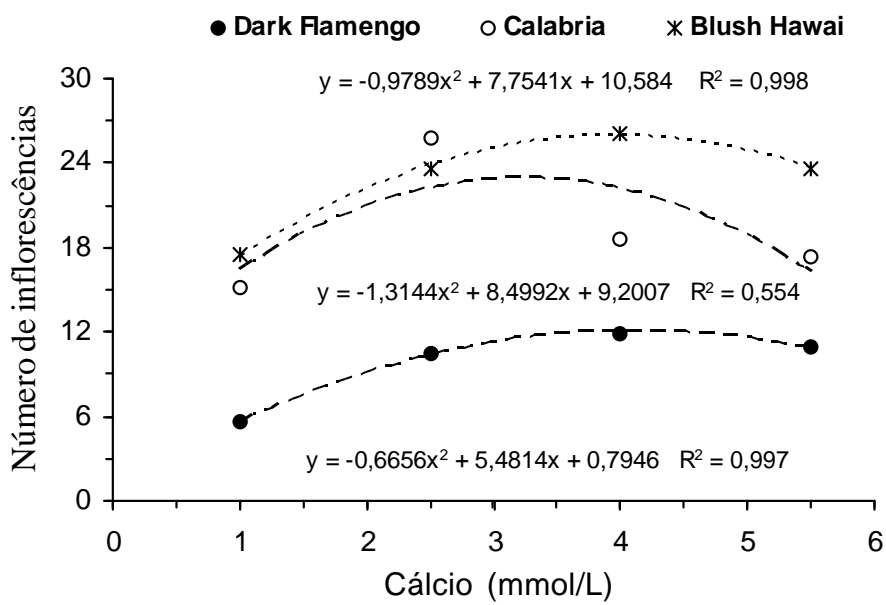

Figura 2 - Número de inflorescências de diâmetro entre 1 e $3 \mathrm{~cm}$ de variedades de crisântemos cultivados em sistema hidropônico em função da aplicação de doses de cálcio.

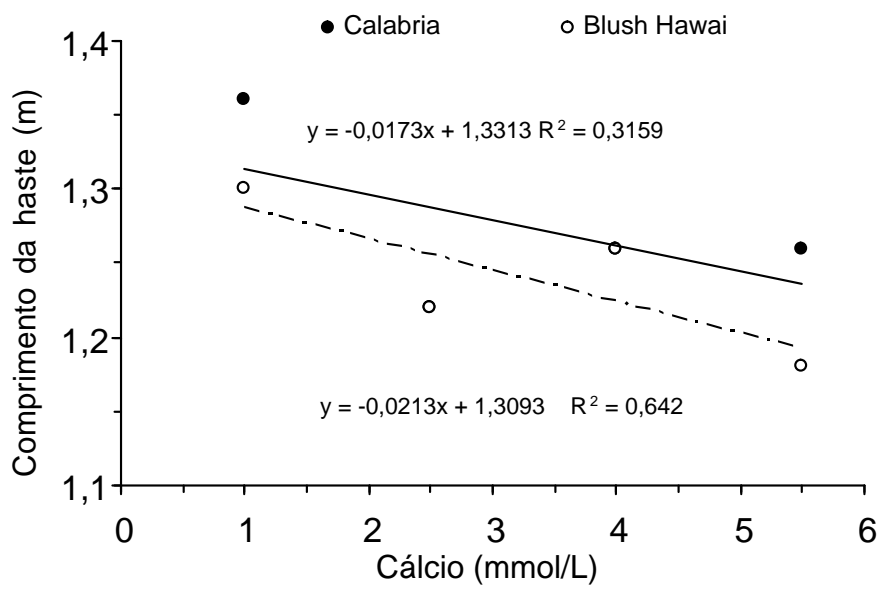

Figura 3 - Comprimento de haste de variedades de crisântemos cultivados em sistema hidropônico em função da aplicação de doses de cálcio.

Tabela 3 - Valores médios para longevidade de variedades de crisântemos cultivados em sistema hidropônico para diferentes doses de cálcio.

Longevidade (dias)

\begin{tabular}{lcccc}
\cline { 2 - 4 } \multicolumn{1}{c}{ Variedades } & \multicolumn{4}{c}{ Doses de Cálcio $\left(\mathrm{mmol} \mathrm{L}^{-1}\right)$} \\
\cline { 2 - 4 } & 1,0 & 2,5 & 4,0 & 5,5 \\
\hline Dark Flamengo & $15,33 \mathrm{a}$ & $16,33 \mathrm{a}$ & $16,00 \mathrm{~b}$ & $16,67 \mathrm{~b}$ \\
Calabria & $16,00 \mathrm{a}$ & $17,00 \mathrm{a}$ & $18,67 \mathrm{a}$ & $19,00 \mathrm{a}$ \\
Blush Hawai & $15,67 \mathrm{a}$ & $16,33 \mathrm{a}$ & $18,33 \mathrm{a}$ & $20,00 \mathrm{a}$ \\
\hline
\end{tabular}

Médias seguidas da mesma letra na coluna, não diferem entre si pelo teste de Newman keuls, $5 \%$. 


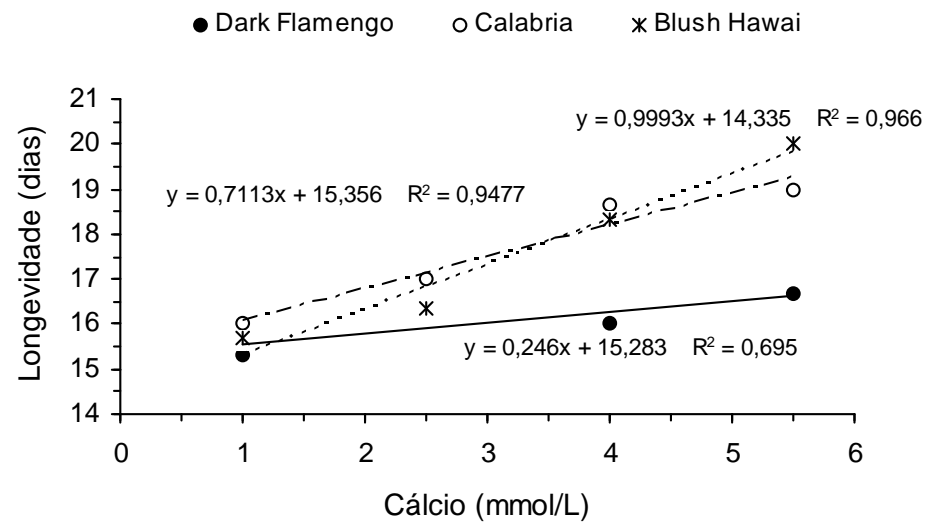

Figura 4 - Longevidade de inflorescências de variedades de crisântemos cultivados em sistema hidropônico em função da aplicação de doses de cálcio.

\section{CONCLUSÕES}

As variedades Calabria e Blush Hawai apresentaram-se mais precoces e mais produtivas em relação à Dark Flamengo. Houve aumento linear na longevidade das inflorescências das três variedades com o aumento das concentrações de cálcio na solução nutritiva. O melhor desenvolvimento e crescimento das variedades de crisântemos foram obtidos quando utilizada a solução nutritiva com $4,0 \mathrm{mmol} \mathrm{L}{ }^{-1}$ de cálcio.

\section{REFERÊNCIAS BIBLIOGRÁFICAS}

BARBOSA, J.G. Crisântemos: produção de mudas, cultivo para corte de flor, cultivo em vaso e cultivo hidropônico. Viçosa, MG: Aprenda Fácil, 2003. 220p.

BARBOSA, J.G.; KAMPF, A.N.; MARTINEZ, H.E.P.; KOLLER, O.C.; BOHNEN, H. Chrysanthemum cultivation in expanded clay: effect of the nitrogen-phosphorouspotassium ratio in the nutrient solution. Journal of Plant Nutrition, London, v.23, n.9, p.1327-1336, 2000.

BRASIL. Ministério da Agricultura, Pecuária e Abastecimento. Brasília, 2008. Disponível em: <http:// 'Www.agricultura.gov.bur>. Acesso em: 16 jun. 2008.

GANMORE-NEUMANN, R.; DAVIDOV, S. Uptake and distribution of calcium in rose plantlets as affected by calcium and boron concentration in culture solution. Plant and Soil, The Hague, v.155/156, p.151-154, 1993.

GISLEROD, H.R. The role of calcium on several aspects of plant and flower quality from a floricultural perspective. Acta Horticulturae, Amsterdam, v.481, p.345-352, 1999.
HALEVY, A.H.; TORRE, S.; BOROCHOV, A.; PORAT, R. Calcium in regulation of postharvest life of flowers. Acta Horticulturae, Amsterdam, v.543, p.345-341, 2001.

HANSEN, R. Chrysanthemums grown in hydroponics: toward development of a cost effective, automated production system. Acta Horticulturae, Amsterdam, v.481, p.297-304, 1999.

LANDGRAF, P.R.C.; PAIVA, P.D. de O. Produção de flores cortadas no estado de Minas Gerais. Ciência e

Agrotecnologia, Lavras, v.33, n.1, p.120-126, jan./fev., 2009.

MUNIZ, M.A.; BARBOSA, J.G.; GROSSI, J.A.S.; ORBES, M.Y.; SÁ, P.G. Produção e qualidade de crisântemos de vaso fertirrigados com diferentes relações nitrato/amônio. Bioscience Journal, Uberlândia, v.25, p.75-82, 2009.

NIELSEN, B.; STARKEY, K.R. Influence of production factors on postharvest life of potted roses. Postharvest Biology and Technology, Amsterdam, v.16, p.157-167, 1999.

ROUDE, N.; TERRIL, A.N.; BARRET, E. Longevity of potted chrysanthemum at nitrogen and potassium concentration and $\mathrm{NH}_{4}: \mathrm{NO}_{3}$ ratios. HortScience, Alexandria, v.26, n.2, p.163-165, 1991.

SILVEIRA, R.B.A.; MINAMI, K. Qualidade de crisântemos (dendranthema grandiflora tzvelev) produzidos em diferentes regiões do estado de São Paulo: Grupo polaris. Scientia Agricola, Piracicaba, v.56, n.2, p.337-348, 1999.

TAIZ, L.; ZEIGER, E. Fisiologia vegetal. 3.ed. Porto Alegre: Artmed, 2003. 719p. 